\title{
Research Paper \\ Effects of Physical Fitness Exercise, Mental Exercise and Mindfulness Exercise on Static and Dynamic Balance in Elderly Women
}

\author{
*Latifeh Ghasempour ${ }^{1}$, Fatemeh Sadat Hoseini ${ }^{1}$, Mehran Soleimani $^{2}$, Malek Ahmadi ${ }^{3}$
}

1. Department of Motor Behavior and Sport Management, Faculty of Sport Science, UrmiaUniversity, Urmia, Iran.

2. Department of Psychology, Faculty of Education and Psychology, Azarbaijan Shahid Madani University, Tabriz, Iran.

3. Department of Physical Education and Sport Sciences, Young Researchers and Elite Club, Urmia Branch, Islamic Azad University, Urmia, Iran.

Received: 03 Feb. 2017 Accepted: 08 May 2017

Keywords:

Physical exercise, Mindfulness,

Mental training, Static and dynamic balance, Elderly women
Cftation: Ghasempour L, Hoseini FS, Soleimani M, Ahmadi M. [Effects of Physical Fitness Exercise, Mental Exercise and Mindfulness Exercise on Static and Dynamic Balance in Elderly Women (Persian)]. Iranian Journal of Ageing. 2017; 12(2):180-191. http:// dx.doi.org/10.21859/sija-1202180

http://dx.doi.org/10.21859/sija-1202180

\begin{abstract}
A B S T R A C T
Objectives Elderly are exposed to balance dysfunction. This research aims at investigating the effect of physical exercises, mindfulness and mental training on static and dynamic balance in elderly women. Methods \& Materials The participants included 60 women in the age group of 60-75 years who were categorized into four groups randomly (three experimental groups "mindfulness, mental training, and physical exercises" and a control group, with 15 members in each group. To measure dynamic and static balance, Berg Balance Scale and Sharpened Romberg test were used. Experimental groups took part in physical exercise and mental training and mindfulness training sessions for eight weeks. Each week had three sessions, and each session was of 75 minutes. Then the post-test was performed, and eight weeks after the post-test, a follow-up test was conducted. For data analyses, the variance for repeated measures and ANOVA test were used at 0.05 significant level.

Results In the post-test balance scores (static and dynamic), there was no significant difference between mindfulness training and mental training groups $(P<0.001)$. But the effects of mindfulness and mental training as compared to physical exercise and control groups were positive and significant $(P<0.001)$. Besides, the effects of training in the physical exercise group were positive and significant compared to control group $(\mathrm{P}<0.001)$.

In the follow-up test balance scores (static and dynamic), there was no significant difference between mindfulness training and mental training groups $(P>0.05)$. Also, there was no significant difference between physical exercise and control groups. However, in comparison to physical exercise and control groups, the effects of mindfulness training and mental training were found to be positive and significant $(\mathrm{P}<0.001)$.

Conclusion The study showed that physical exercises, mindfulness and mental training have a significant effect on the improvement of static and dynamic balance in elderly women, though the association of cognitive practices (mindfulness and mental training) with motor training (physical exercises) could cause a lasting impact. So, it is recommended to use physical exercises with cognitive training to improve static and dynamic balance in elderly people.
\end{abstract}

\section{* Corresponding Author:}

Latifeh Ghasempour, PhD

Address: Department of Motor Behavior and Sport Managment, Faculty of Sport Science, Urmia University, Urmia, Iran.

Tel: +98 (914) 4484693

E-mail: ghasempourlatifeh@yahoo.com 


\section{Extended Abstract}

\section{Objectives}

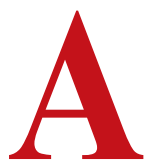

geing is a process that encompasses all human existence through getting older based on gradual and progressive changes. Most of the adults, as they become older, show a substantial drop in motor ability. In the course of ebb in motor ability of adults, their ability to preserve balance would be reduced too. Unfortunately, half of the elderly who fall, no longer walk normally; undoubtedly, the number of falling could be decreased among the elderly. One of the most important strategies for reducing falling among the elderly is maintaining a physically active lifestyle. A physical fitness program compensates the decline in such areas. The study aimed at analyzing the effects of physical fitness exercise, mental exercise and mindfulness exercise on static and dynamic balance in elderly women.

\section{Methods \& Materials}

The elderly who satisfied the research criteria (female, within the age range of 60-75-years, without any cognitive problems, lack of regular sportive and physical activity, without any disease in the past [suffering from no diseases such as neuromuscular, orthopedic, fracture, structural defect, cardiovascular diseases, metabolic], no experience in mental courses [such as mental exercise, imagery, and mindfulness courses] until performing the test, lack of motor limitation, lack of using any effective neuro medications on balance) participated in the study voluntarily. They gave their consent for tests and practice sessions. They were sub-divided into four groups (three experimental groups of mental exercise, mindfulness exercise and physical fitness exercise, and one control group). Each group consisted of 15 persons. Balance Tests of Berg (for measuring dynamic balance), Sharpened Romberg (for measuring static balance), a shortened cognitive questionnaire, and a demographic questionnaire including information like age, having any disease in the past, experience of falling, experience of participation in a sport program, and consuming drugs were used.

The experimental groups participated in exercises for eight weeks (three 75 minutes sessions per week) in terms of the following protocol: Physical fitness group did only physical fitness exercises throughout the exercise regime; Mental exercise group, in addition to physical fitness exercises, practiced mental exercise that lasted for 15 minutes in each session; Mindfulness group did physical fitness exercises in two sessions in a week and mindfulness exercise in one session in a week; Control group did not participate in any of the exercise sessions during experimental group exercises.

After the end of the exercise period, post-test was performed, and after eight weeks, a follow-up test were performed. For data analysis, repeated measures ANOVA test and One-Way ANOVA with Sig. $=0.05$ were applied.

\section{Results}

Age mean and standard deviation of the participated groups in the study are as follows: Mental Exercise Group (mean $=69.25$ years, $S D= \pm 4.33$ ), Mindfulness $E x-$ ercise Group (mean $=72.23$ years, $\mathrm{SD}= \pm 3.00$ ), Physical Fitness Exercise Group (mean=70.34 years, $\mathrm{SD}= \pm 3.68$ ), and Control Group (mean $=67.65$ years, $S D= \pm 5.55$ ). Kolmogorov-Smirnov test results showed normal distribution of variables $(\mathrm{P}>0.05)$, and it was suitable for doing parametric statistics tests. Result of analyzing sphericity assumption showed that the assumption was not proved

Table 1. Mean and standard deviation of the participant scores in components of static and dynamic balance in 3 steps of the test

\begin{tabular}{|c|c|c|c|c|c|}
\hline Groups & Tests & 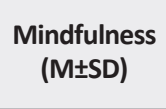 & $\begin{array}{l}\text { Mental Exercise } \\
\text { (M士SD) }\end{array}$ & 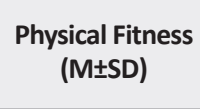 & $\begin{array}{l}\text { Control } \\
(\mathrm{M} \pm \mathrm{SD})\end{array}$ \\
\hline \multirow{3}{*}{$\begin{array}{c}\text { Sharpened } \\
\text { Romberg }\end{array}$} & Pre-test & $20.93 \pm 1.91$ & $20.00 \pm 2.73$ & $20.27 \pm 2.76$ & $19.07 \pm 1.58$ \\
\hline & Post-test & $11.67 \pm 1.59$ & $11.73 \pm 1.87$ & $15.00 \pm 2.45$ & $18.40 \pm 1.07$ \\
\hline & Follow-up test & $14.27 \pm 1.44$ & $14.60 \pm 1.96$ & $17.67 \pm 2.69$ & $18.07 \pm 1.39$ \\
\hline \multirow{3}{*}{$\begin{array}{l}\text { Berg Balance } \\
\text { Scale }\end{array}$} & Pre-test & $31.00 \pm 2.42$ & $31.13 \pm 1.81$ & $32.00 \pm 2.10$ & $32.33 \pm 1.88$ \\
\hline & Post-test & $46.87 \pm 1.80$ & $46.60 \pm 1.55$ & $39.8 \pm 2.45$ & $33.4 \pm 1.84$ \\
\hline & Follow-up test & $43.13 \pm 2.33$ & $43.47 \pm 2.17$ & $35.6 \pm 2.26$ & $33.87 \pm 1.96$ \\
\hline
\end{tabular}


$(\mathrm{P}<0.05)$. Therefore, in analyzing data of Repeated Measures test Greenhouse-Geisser's correction to the degrees of freedom was used. Descriptive statistics are presented in Table 1.

Then repeated measures ANOVA for analysis balance (static and dynamic) was applied, and results showed that testing effect, group, and interaction of testing effect and group was significant $(\mathrm{P}<0.001)$. However, as the test did not show in which step and among which groups the difference was significant, the ANOVA test in three steps of the test (pre-test, post-test and follow-up test) was used for obtaining precise results. Findings of the research on improving static and dynamic balance in the elderly women who participated in physical fitness exercises, mindfulness, and mental exercise are as follows.

Mindfulness exercise and mental exercise had a positive and significant effect on static balance in post-test and follow-up stages $(\mathrm{P}<0.001)$, and there were no significant differences between these two types of exercises in terms of effectiveness and durability in post-test and follow-up steps $(\mathrm{P}>0.05)$. However, physical fitness exercises had just positive and significant effect on static balance in post-test step ( $\mathrm{P}<0.001)$. As well as, effect of mindfulness exercise and mental exercise on static balance was positive and significant in comparison with physical fitness exercise and control in post-test and follow-up test $(\mathrm{P}<0.001)$

Mindfulness exercise and mental exercise also had a positive and significant effect on dynamic balance in posttest and follow-up stages $(\mathrm{P}<0.001)$, and there was no any significant difference between these two types of exercises in terms of effectiveness and durability in post-test and follow-up stages $(\mathrm{P}>0.05)$. However, physical fitness exercises had a positive and significant effect on dynamic balance in post-test stage $(\mathrm{P}<0.001)$. The effect of mindfulness exercise and mental exercise on dynamic balance, in comparison with physical fitness exercise and control, was positive and significant in post-test and follow-up test $(\mathrm{P}<0.001)$

\section{Conclusion}

Results showed that physical fitness, mindfulness and mental exercises had an effect on improving static and dynamic balance in the elderly women; the advantages of the three kinds of exercises could be applied for improving balance of this group of society. However, doing mental and mindfulness exercises with physical fitness exercises caused creating permanence impact of exercises on static and dynamic balance. In this regard, a combination of cognitive exercises (mental and mindfulness exercises) and motor exercise (physical fitness exercises) caused better and more stable results for the research samples.

Therefore, it is recommended that: 1) All coaches and therapists of sports, rehabilitation and training centers should use all benefits of the three methods in order to improve the balance of the elderly who need to strengthen their balance, according to physical and cognitive characteristics of the elderly; and 2) Researchers should use exercise protocol of the study for treatment of various physical injuries in the elderly.

\section{Acknowledgments}

This paper is extracted from the $\mathrm{PhD}$ dissertation of the first author in the Department of Motor Behavior and Sport Managment, Faculty of Sport Science, UrmiaUniversity, Urmia.

\section{Conflict of Interest}

The authors declared no conflicts of interest. 


\title{
تأثير تمرينهاى أمادتى جسمانى و تمرين ذهنى و تمرين ذهن أكاهى بر تعادل ايستاو يوياى زنان سالمند
}

\author{
"طيفه قاسميور'، فاطمه سادات حسينى'، مهران سليمانى"، مالك احمدى" \\ ا- كروه رفتار حركتى و مديريت ورزشى، دانشكده علوم ورزشى، دانشعاه اروميه، اروميه، ايران.

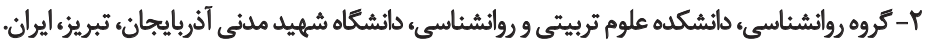

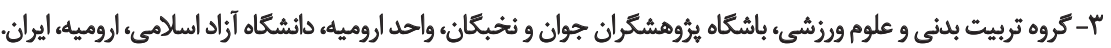

\begin{abstract}
حكبد

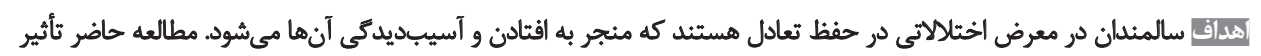

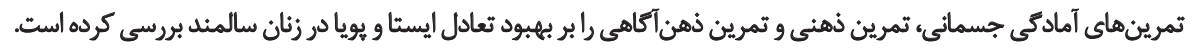

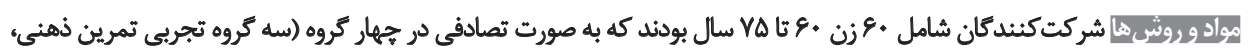

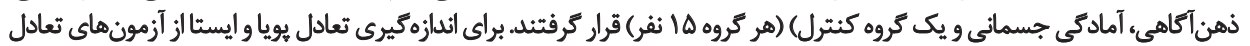

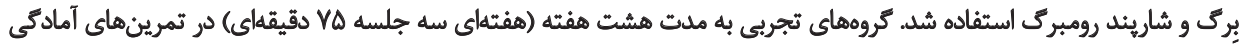

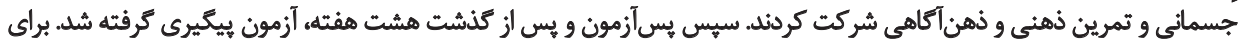

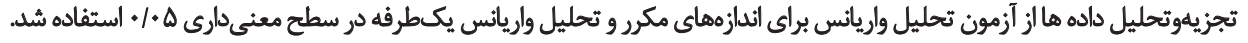

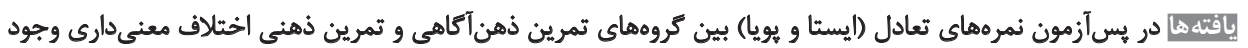

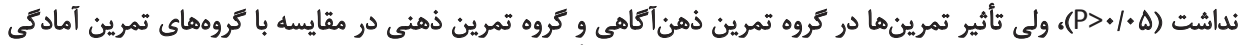

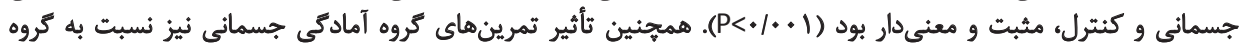

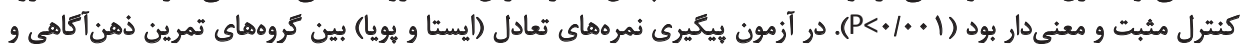

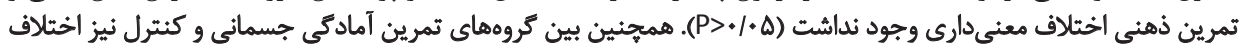

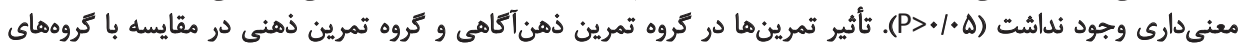

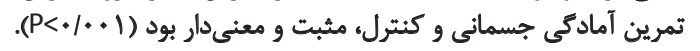

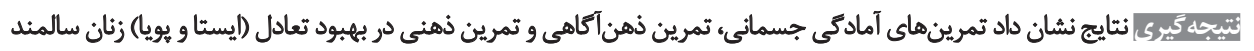

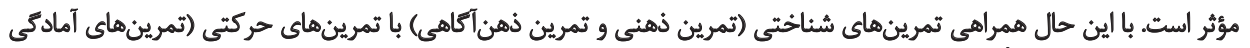

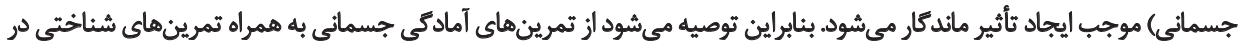

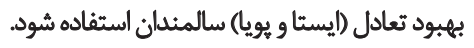

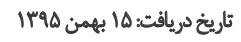

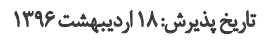

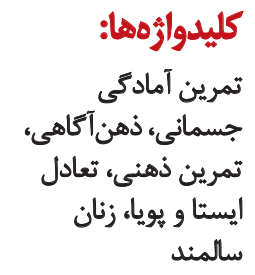

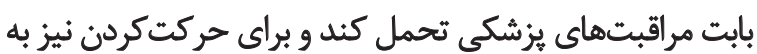

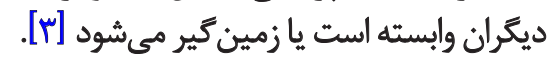

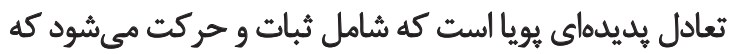

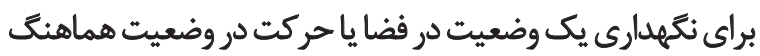

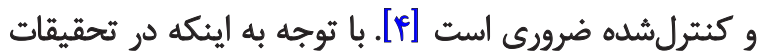

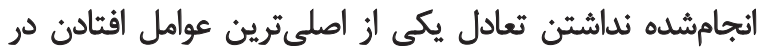

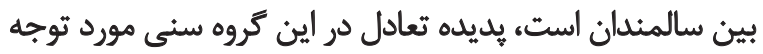

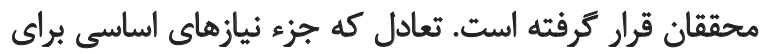

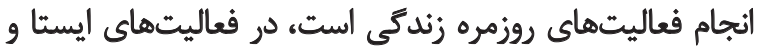

dates سالمندى فرايندى است كه بر اساس تغييرات آرام و بيشرونده

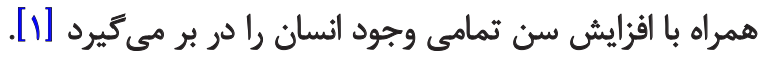

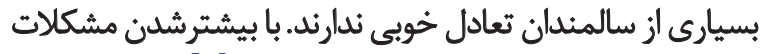

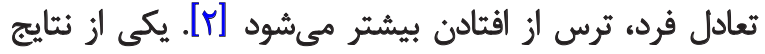

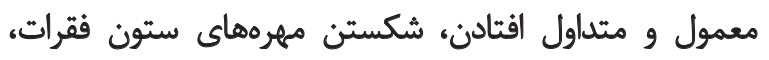

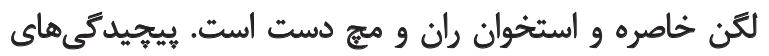

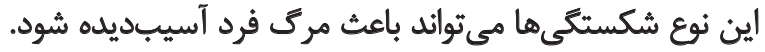

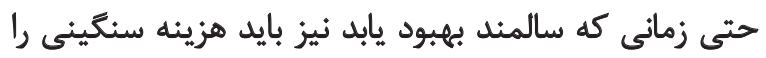




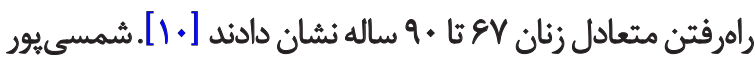

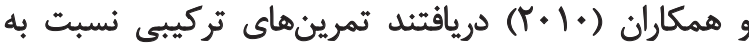

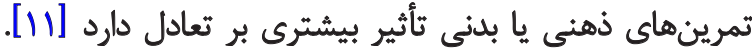

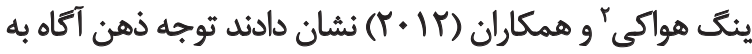

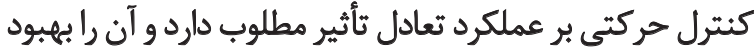

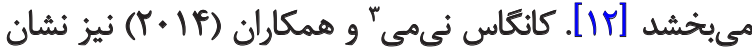

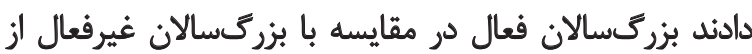

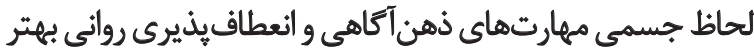

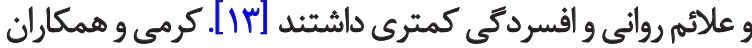
(T P / If)

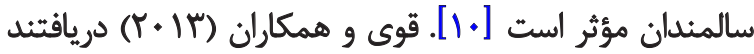

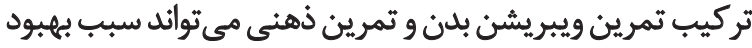

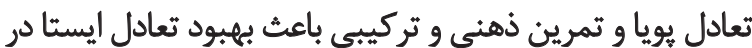

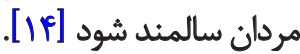

حسينى (1ل (T) دريافت بهبود وضعيت تعادل در تروه تمرين

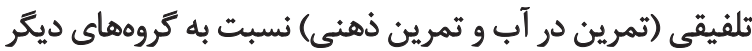

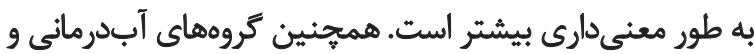

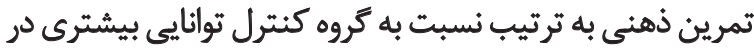

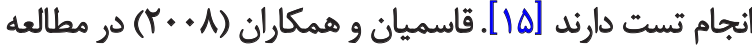

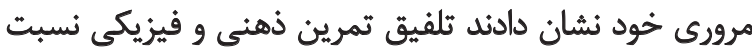

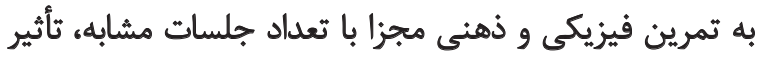

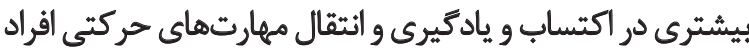

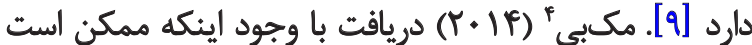

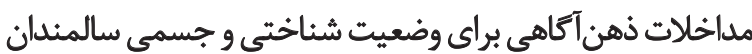

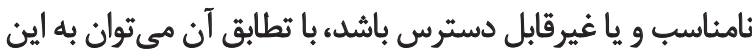

اهداف دست يافت.

البته مطالعات اندكى آموزش ذهن آكاهى سالمندان را هدف

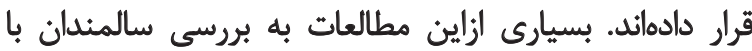

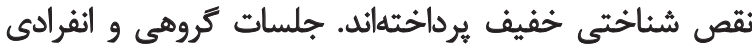

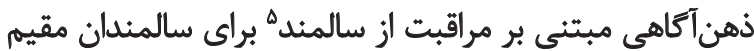

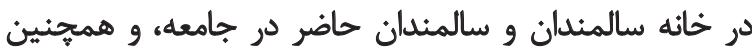

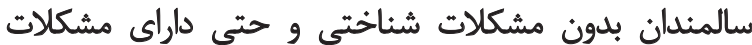

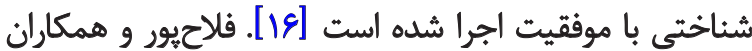

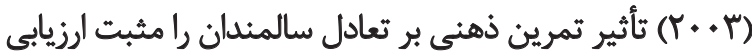

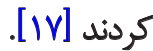

مرور مطالعات انجامشده در زمينه سالمندان و عوامل مؤثر بر مير

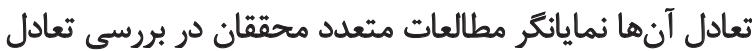

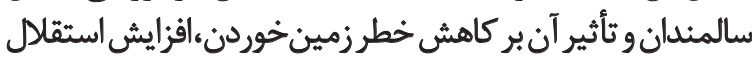

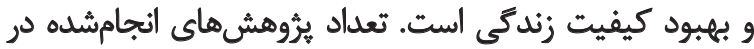

\section{Ying Hwakee}

3. Kangasniemi

4. McBee

5. Mindfulness Based Elder Care (MBEC)

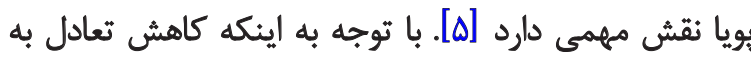

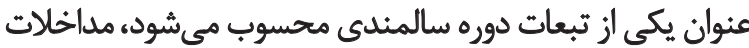

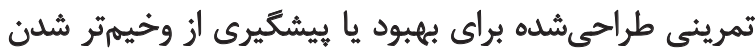

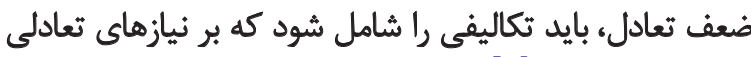
تمركز داشته باشد [ه]. مهارتهاى حركتى و روائى در تعامل با يكديكر وضعيت فرد

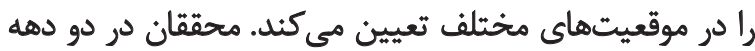

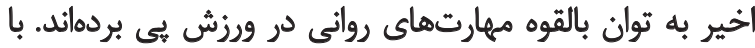

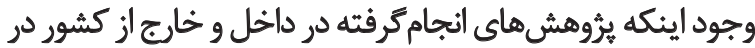

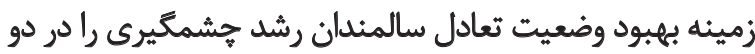

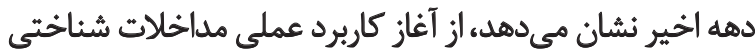

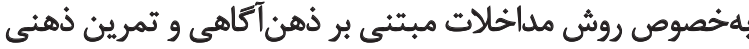

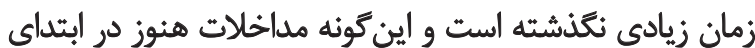

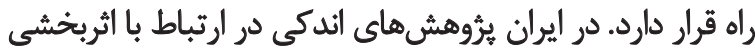

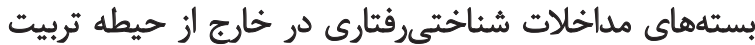

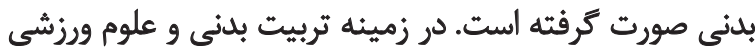

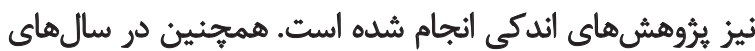

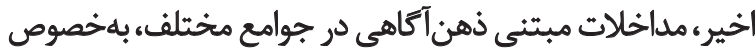

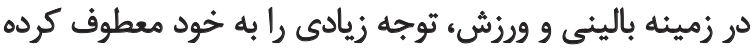

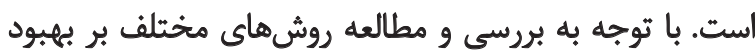

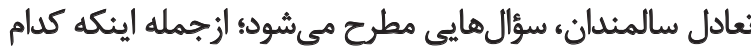

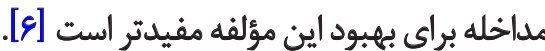
ذهنآكاهى به معنى توجهكردن به زمان حال به صورت

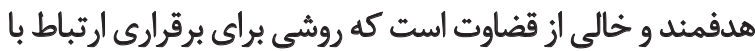

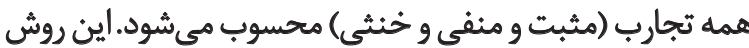

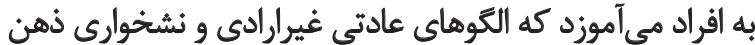

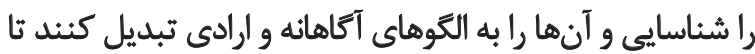

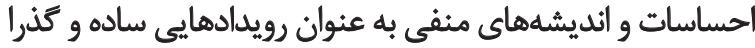

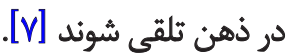

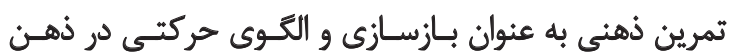

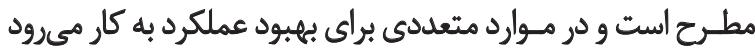

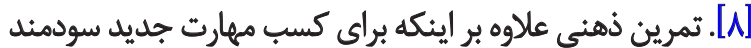

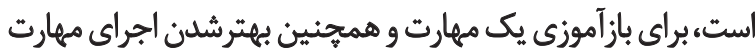

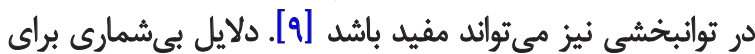

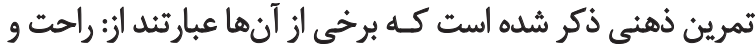

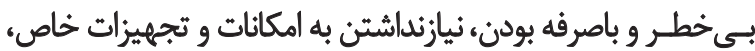

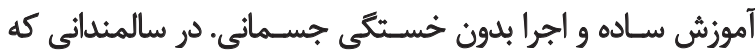

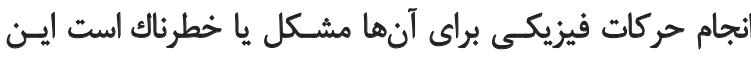

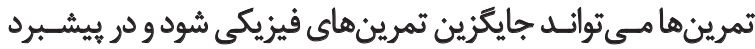

اهداف توانبخشى مـؤثر باشد [.1]].

ليندن' و همكاران سودمندى تمرين ذهنى را براى بهبود 
دانشكاه اروميه (دانشكده تربيت بدنى و علوم ورزشى) رسيد.

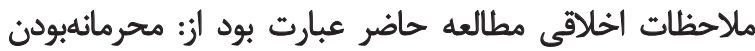

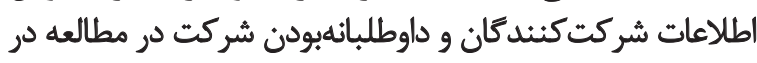

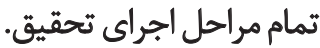

به منظور لحاظكردن موارد ايمنى شركت كنيندكان و اطمينان

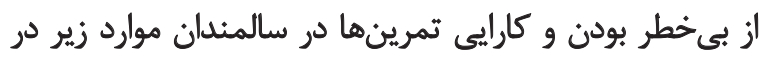

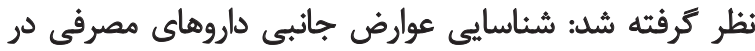

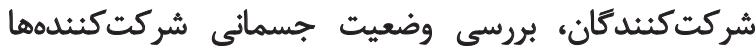

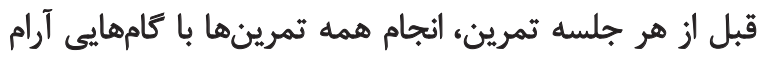

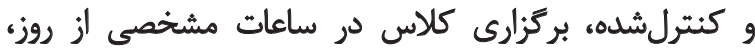

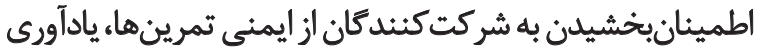

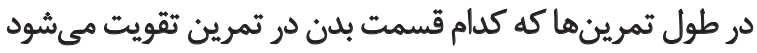

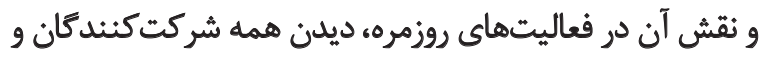

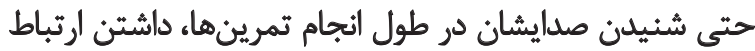

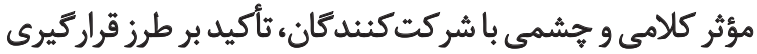

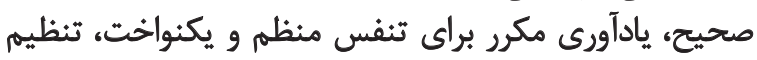

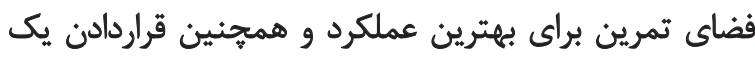

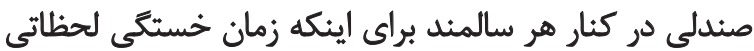

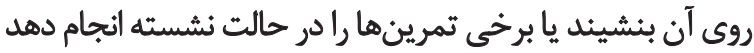

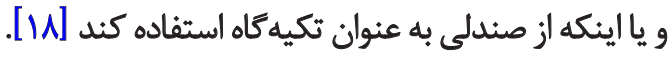

براي ايمنى مكان تمرينها موارد زير در نظر ترفته شد: داشتن

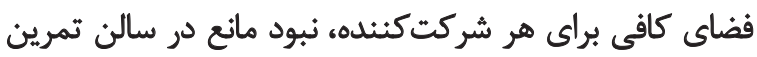

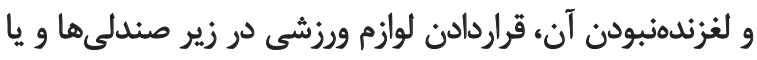

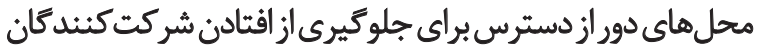

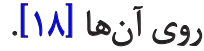

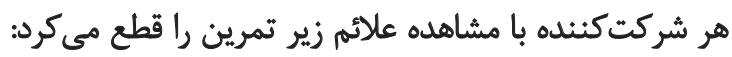

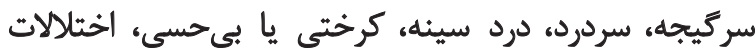

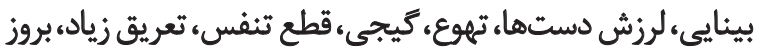

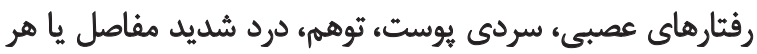

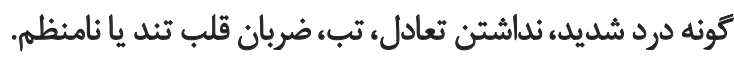
براى شرايط اضطرارى در طول تمرين اقدامات زير انجام

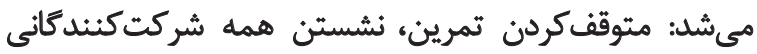

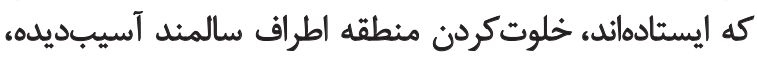

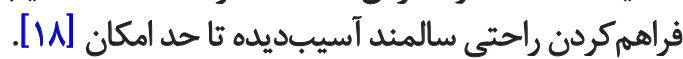

براى بررسى ميزان تعادل ايستا و بويا از نسخه فارسى آزمون

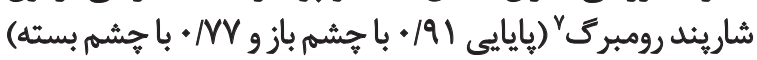

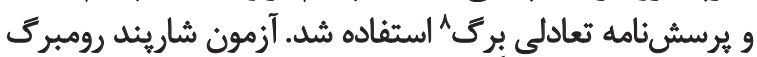

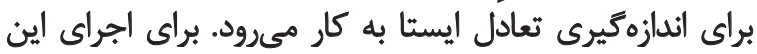

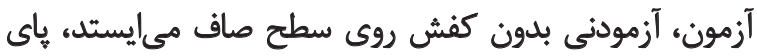

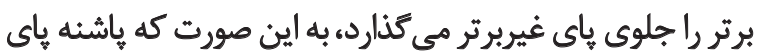

7. Sharpened Romberg

8. Berg Balance Scale (BBS)
زمينه تأثير تمرين ذهنى بر بهبود تعادل بسيار اندك است. نبود

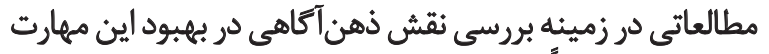

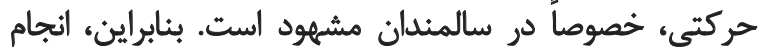
مطالعاتى با رويكرد بررسى تأثير و مقايسه مداخلات شناختي مثاني

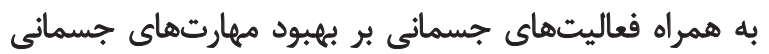

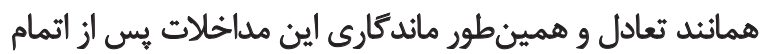

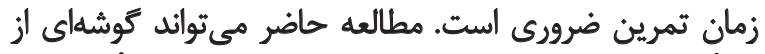

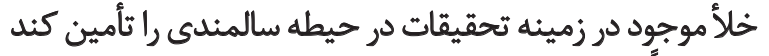

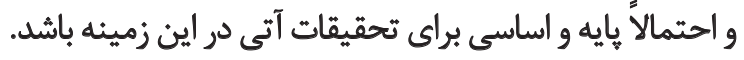

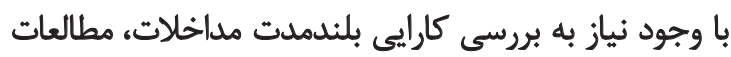

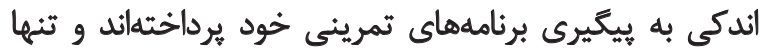

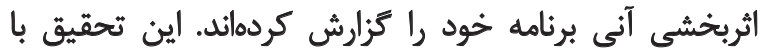

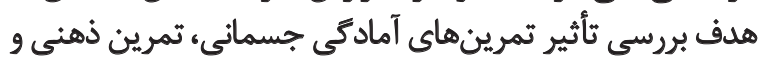

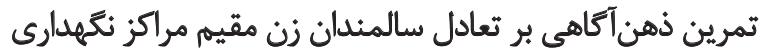

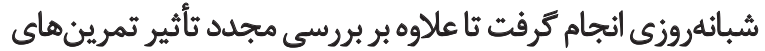

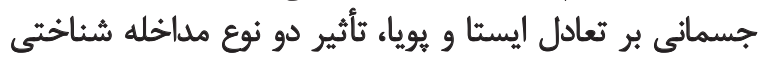

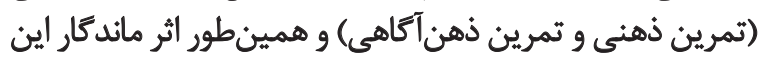

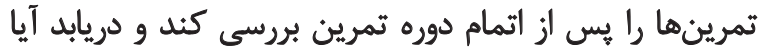

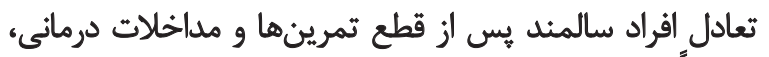

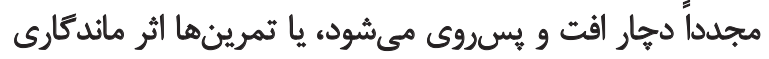

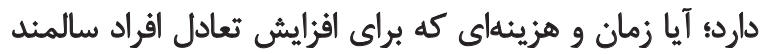

$$
\begin{aligned}
& \text { صرف مى شود تأثيركذار است؟ زئ براي } \\
& \text { روش مطالعه }
\end{aligned}
$$

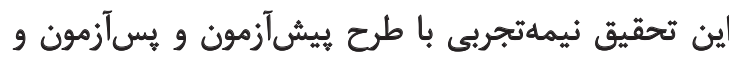

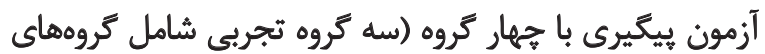

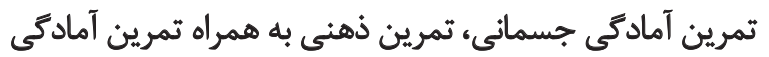

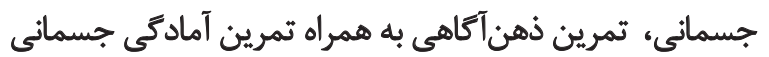

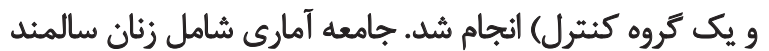

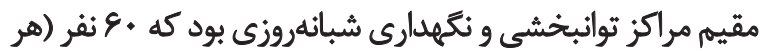

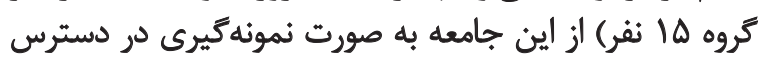

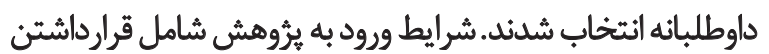

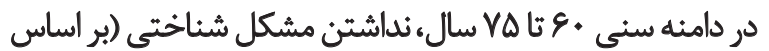

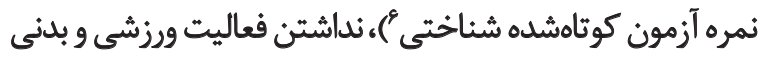

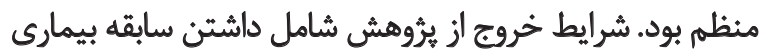

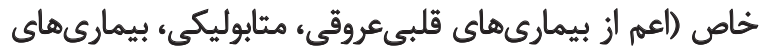

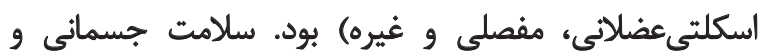

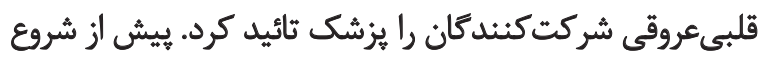

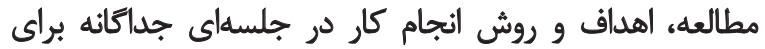

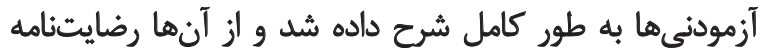

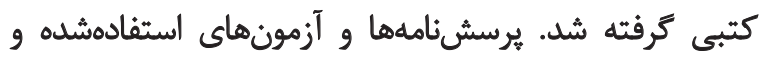

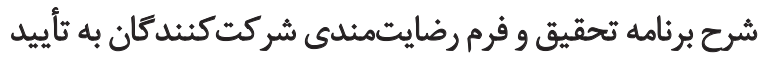


تمركز روى تنفس و بدن و صداء مراقبه راهرفتن، مراقبه نشسته

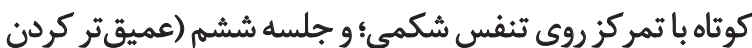

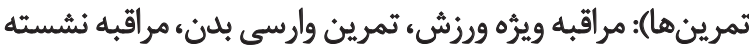

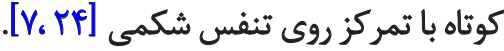

\section{بونامه مداخله تمرين ذذهني}

سالمندان كروه تمرين ذهنى، همراه با تمرينهاي آمادگى

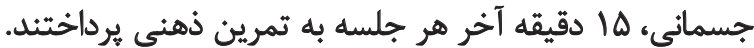

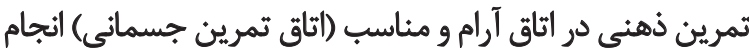

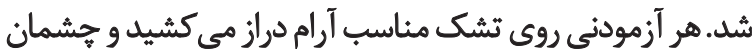

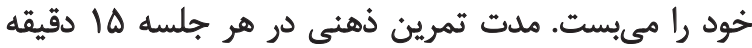

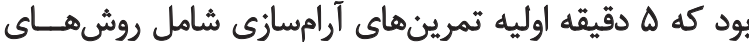

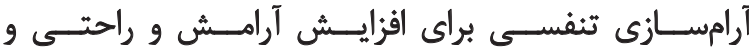

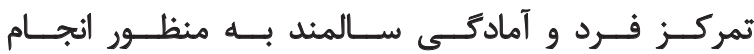

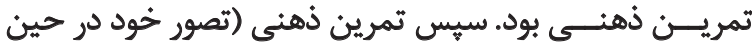

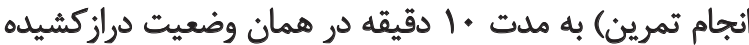

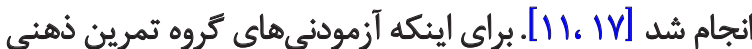

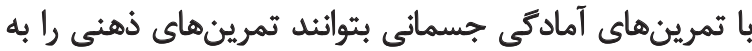

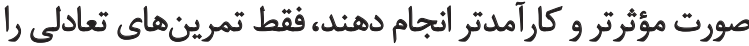
به صورت ذهني انجام دادند.

$$
\text { برنامه تمرين هاى آمادّى جسمانى }
$$

اين تمرينها در جهار بخش (تمرينهاى برخاستن، تمرينهاى

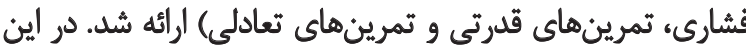

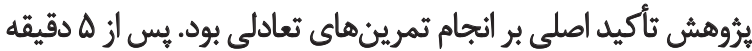

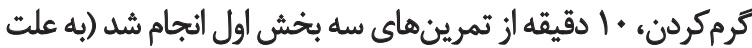

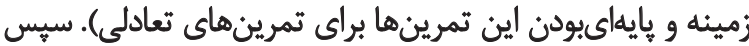

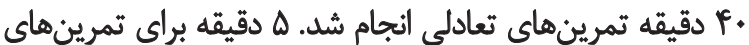

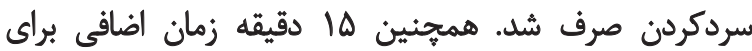

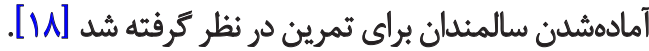

به منظور بررسى تأثير و ماندكارى تمرينها در هر تروها

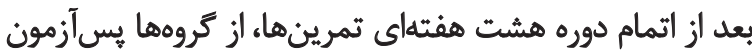

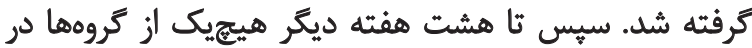

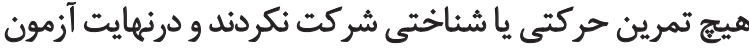

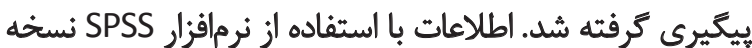

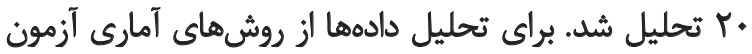

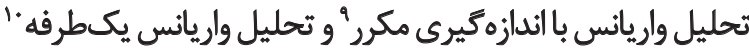

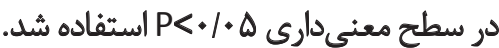

يافتهها

شاخصهاى توصيفى شامل سن، قد و وزن، شاخص توده بدنى

9. Repeated measures 10. ANOVA
جلو به بنجه هياى عقب برخورد كند. دستها به حالت ضربدر

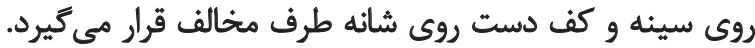

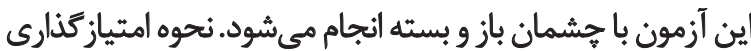

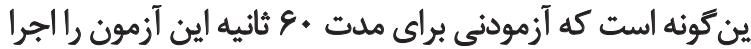

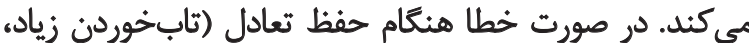

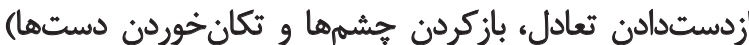

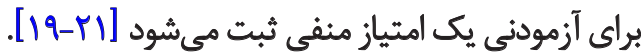
آزمون تعادل بركى براي اندازهيرى تعادل يويا استفاده هي شيود

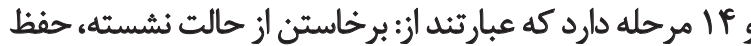

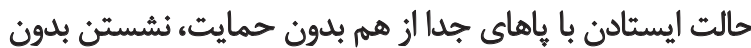

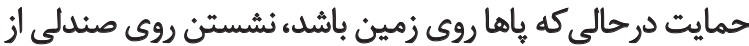

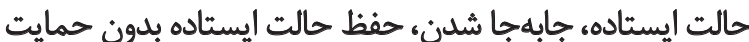

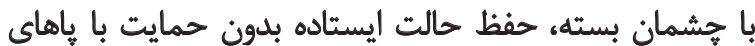

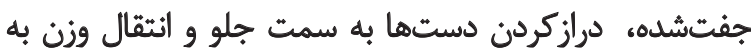

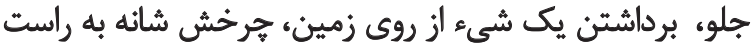

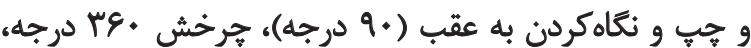

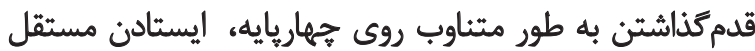

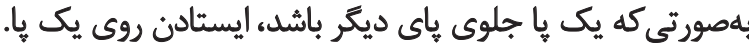

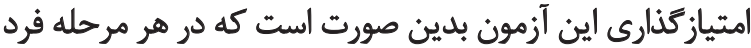

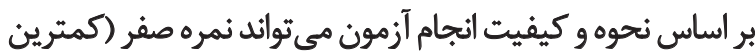

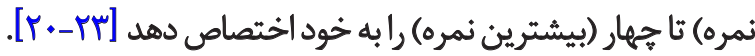

$$
\text { برئاهd مدا/خله مبتنى بر ذهن آتاهى }
$$

در تمرين ذهن آكاهى سالمندان تأ هفته ششم، يك جلسه

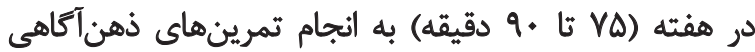

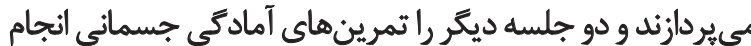

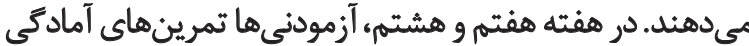

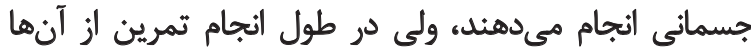

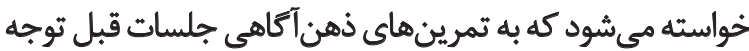

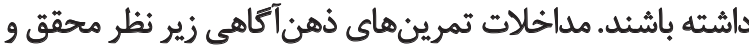

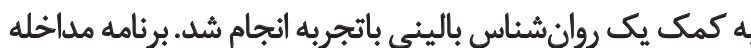

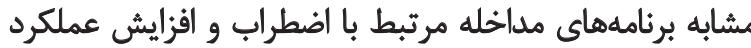

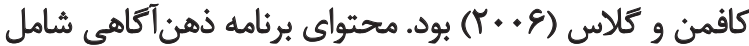

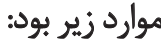

جلسه اول (خوشامدكويى و بيان اهداف): توضيح مفاهيم

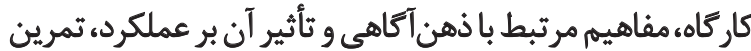

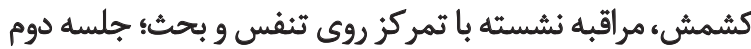

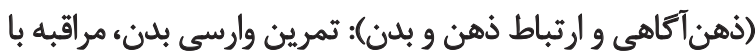

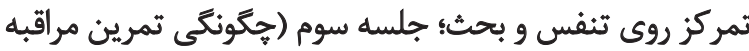

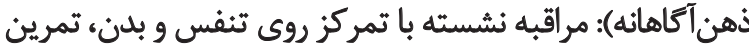

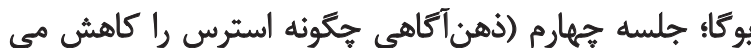

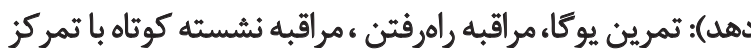

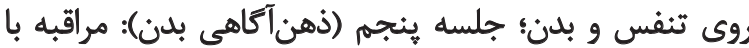




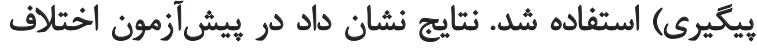

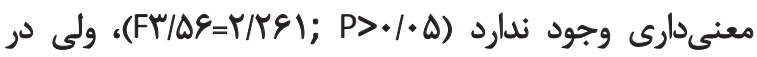

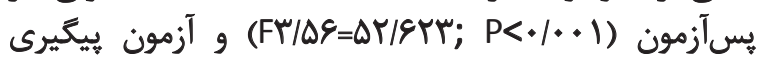

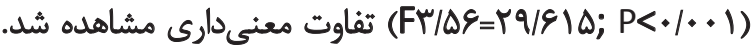

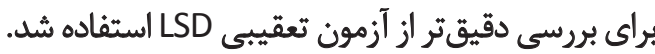

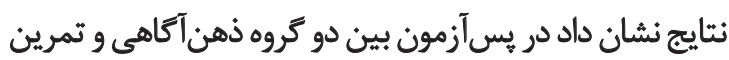

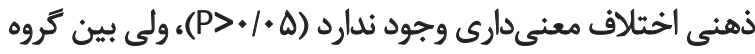

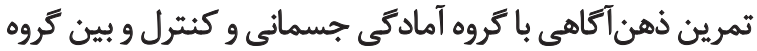

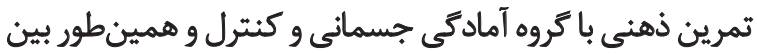

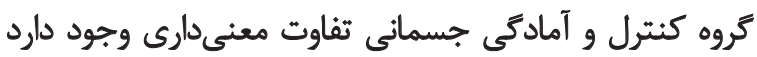

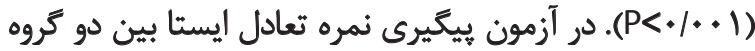

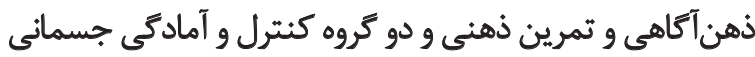

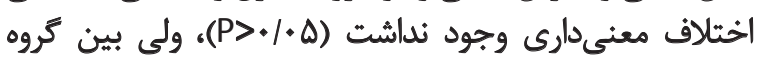

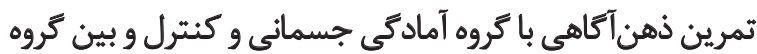

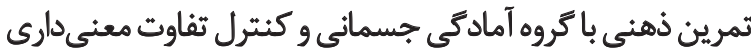

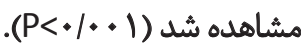

نتايج آزمون تحليل واريانس با اندازهكيرى مكرر تعادل بوريا

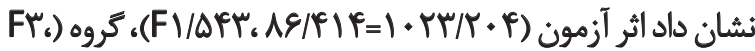

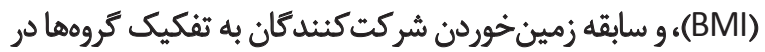

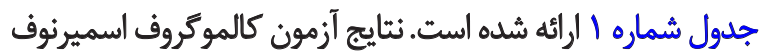

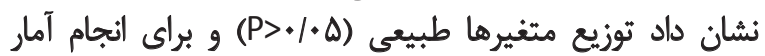

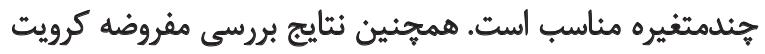

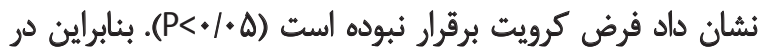

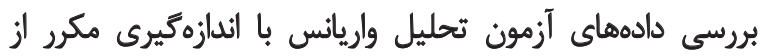

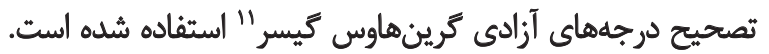

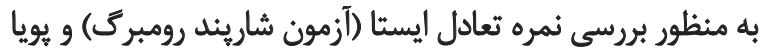

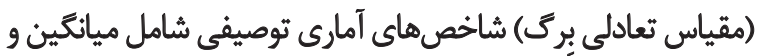
انحرافمعيار در جدول شماره بار ارائه شده است.

براي بررسى تعادل ايستا از آزمون تحليل واريانس با

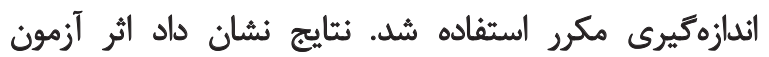

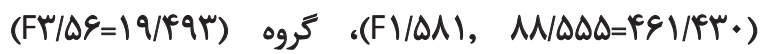
و تعامل اثر آزمون و كروه (FF/NFF=FN/IIT) معنى

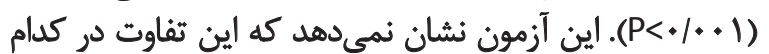

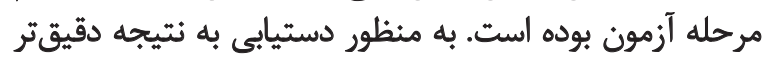

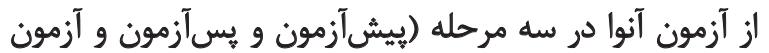

11. Greenhouse-Geisser

جدول (ا شاخصهاى توصيفي كروها

\begin{tabular}{|c|c|c|c|c|}
\hline $\begin{array}{c}\text { كثترل } \\
(M \pm S D)\end{array}$ & 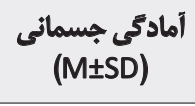 & تمرين ذهنى & ذأنآثاهى & شاخص هاي توصيفى \\
\hline$\varepsilon \vee / \& \Delta \pm \Delta / \Delta \Delta$ & $V \cdot / M \pm \pm r / \varepsilon A$ & $\varepsilon q / T \Delta \pm \psi / \pi T$ & $n / T H \pm r / \cdots$ & سن (سال) \\
\hline$V / \Delta V \pm+1+9$ & $1 / F \lambda \pm=/ / r$ & $1 / 49 \pm+11$ & $1 / P Y \pm+11$ & قد (سائتىمتر) \\
\hline $\mathscr{E} / \mathrm{IV} \pm 1 \mathrm{Q} / \mathrm{T}$ & $\Delta V / \cdot r \pm I g / M$ & PNDQ \pm Q/ET & $\Delta F / A Y \pm I F / F V$ & وزن (كيلوكرم) \\
\hline re/gqtr/qq & $r \Delta / \tau+ \pm \Psi / W$ & $r T / r \Lambda \pm \Psi / \Psi \Lambda$ & $r \varepsilon / T^{c}+ \pm \varepsilon / T Y$ & شاخص توده بدنى (BMI) \\
\hline ع ع عاعو درصد & ل العدصد. & 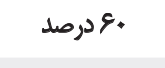 & F & داشتن تحصيلات \\
\hline Dop felfe & ل & س & tojpt. & سابقه زمين خوردن \\
\hline
\end{tabular}

جدول r. ميانكين و انحرافمعيار نمرههاى آزمودنى ها در مؤلفه تعادل ايستا و يويا در سه مرحله آزمون

\begin{tabular}{|c|c|c|c|c|c|}
\hline $\begin{array}{c}\text { كثترل } \\
(M \pm S D)\end{array}$ & 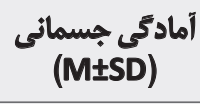 & تمرين ذهنى & (MESD) & تئوهما & أزهونها \\
\hline $19 / \cdot V \pm V / \Delta A$ & $r \cdot / T Y \pm T / N E$ & $r \cdot 1 \cdot . \pm T / M$ & $r \cdot / 9 r \pm 1 / 91$ & ييش آزمون & \multirow{3}{*}{ رومبرى } \\
\hline$W F \cdot \pm 1 / \cdot V$ & $\mid Q / \cdots \pm T / F \Delta$ & $11 M^{\top} \pm 1 / A V$ & $11 / 8 V \pm 1 / \Delta 9$ & يسآزمون & \\
\hline$w \circ V \pm 1 / 7 q$ & $\mid V / \& V \pm r / \& q$ & $14 / 8+ \pm 1 / 98$ & If/TVII/RF & أزمون ييكيرى & \\
\hline$T T / \pi T \pm 1 / M$ & $\pi r / \circ \pm \pm r /$. & $r|/| r \pm|/ A|$ & $r M / . \pm t / A r$ & ييش آزمون & \multirow{3}{*}{ تعادلى برى } \\
\hline$\pi / f \cdot \pm V / A F$ & $r q / \lambda+ \pm r / F \Delta$ & $\mid \forall / 8 \cdot \pm 1 / \Delta \Delta$ & $\forall \& / A V \pm 1 / \Lambda$. & يسآزمون & \\
\hline$M / A v \pm 1 / q$ & $r \Delta / \varepsilon \cdot \pm Y / Y \varepsilon$ & $P T / P V \pm r / I V$ & $r r / N T \pm r / r r$ & آزمون ييكيرى & \\
\hline
\end{tabular}


و يويا مى إشود و تمرينهاى آمادگى جسمانى اثر ماندكارى بر تعادل ايستاو يوياي زنان سالمند ندارد.

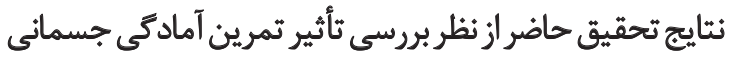

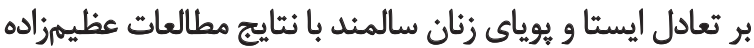

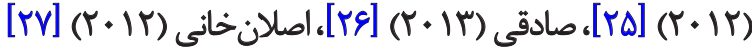

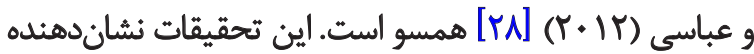

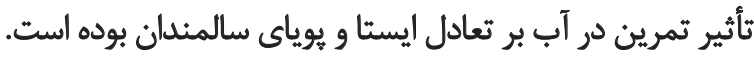

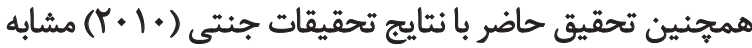

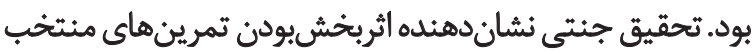

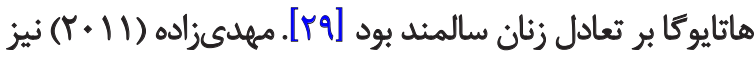

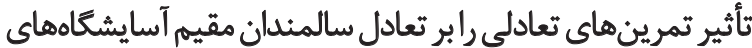

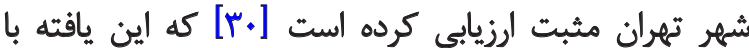
يافتههاى يُروهش بيشرو همسو است.

در مطالعات بيشين تمام تمرينها جسمى بوده و هيج مداخله

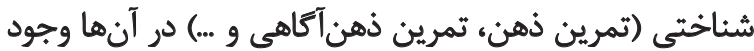

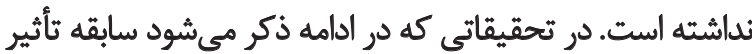

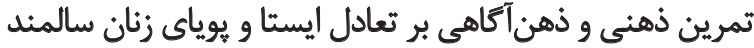

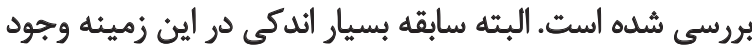

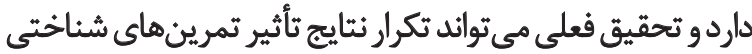
و غناى بيشينه تحقيق در اين زمينه باشدائ نكرائر

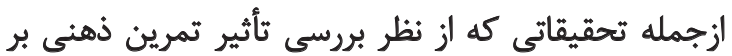

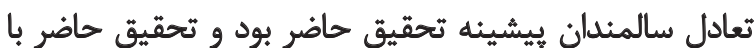

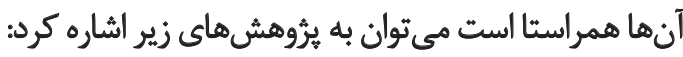
فلاحيور به تأثير مثبت تمرين ذهنى بر تعادل سالمندان اشاره

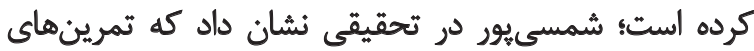

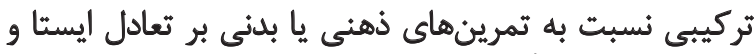

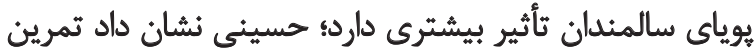

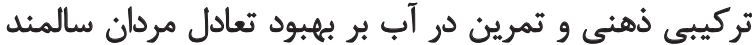

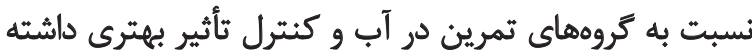

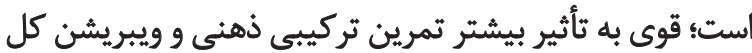

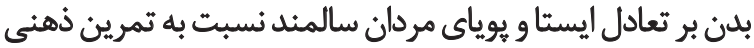

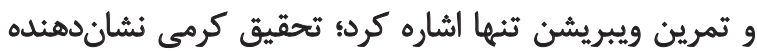

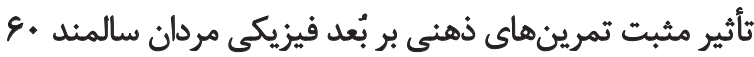

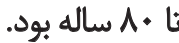

همانطور كه نتايج نشان داد تمرينهاي تركيبى ذهنى و

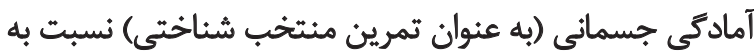

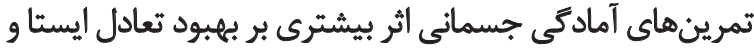

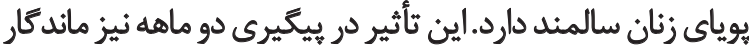

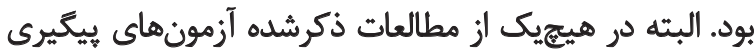

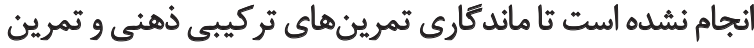

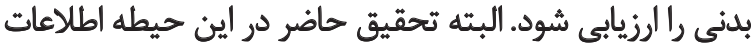
بيشترى به محقق ارائه مي دهد.

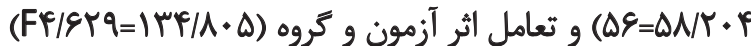

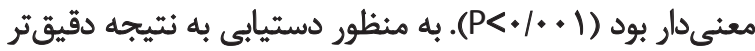

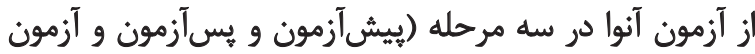

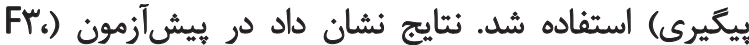

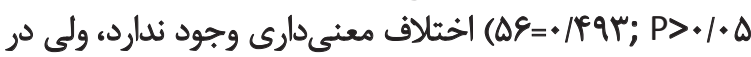

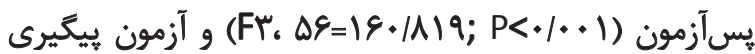
تفاوت معنى دار مشاهده شد. براى بررسى دقيقتر از آزمون تعقيبى LSD استفاده شد. نتايج

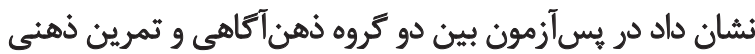

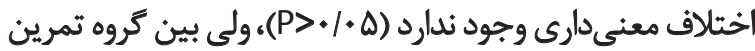

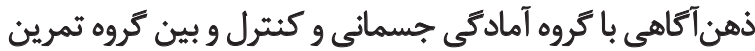

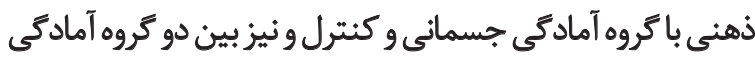

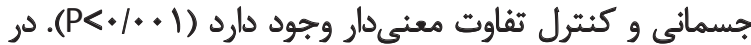

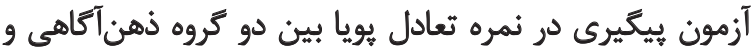

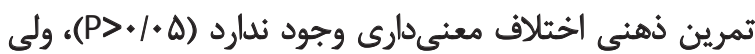

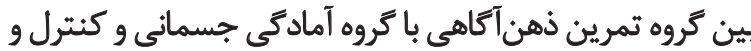

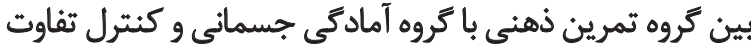

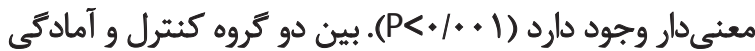

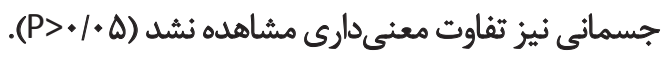

ب

هدف اصلى تحقيق، بررسى تأثير تمرينهاى ذهنى، ذهن آكآهى

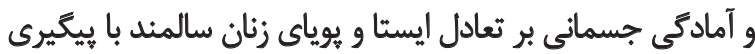

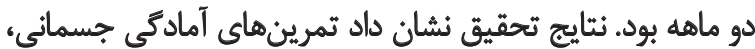

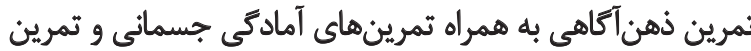

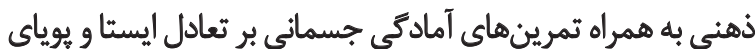

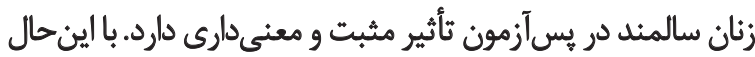

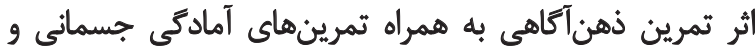

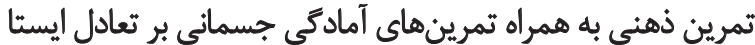

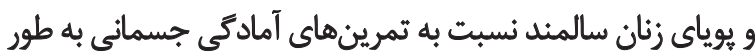

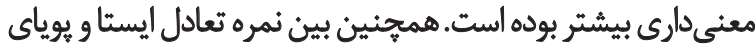

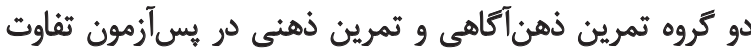

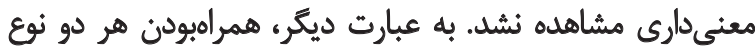

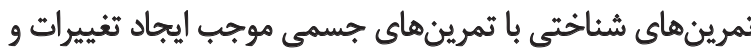

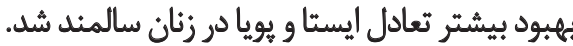
در آزمون بيكيرى فقط تأثير تمرين ذهن آكاهى به همراه

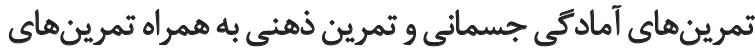

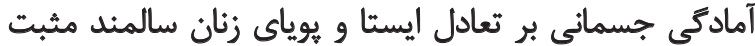

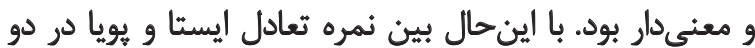

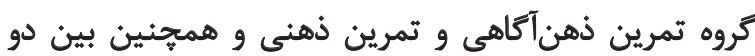

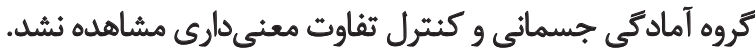

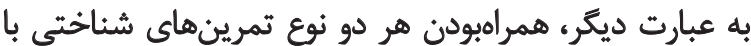

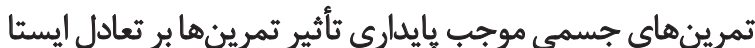


كل بهزيستى استان آذربايجان غربى، مسئولان مراكز نتخهدارى و

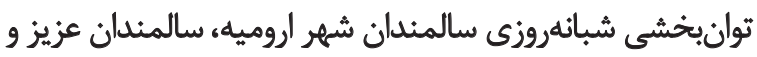

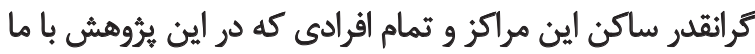
همكارى كردند سياستزارى مى كنيه.

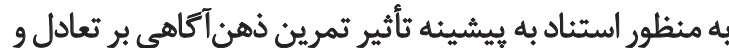

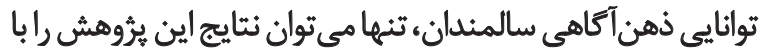

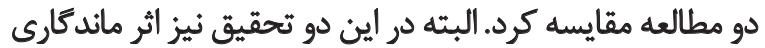

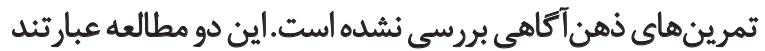

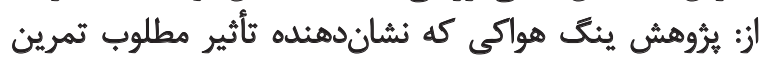

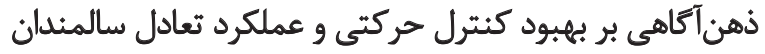

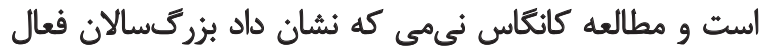

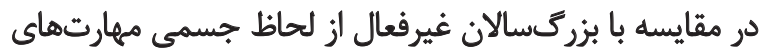

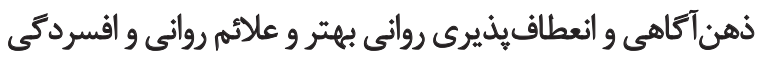

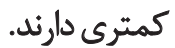

\section{تتيجهَيرى نهايى}

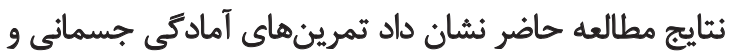

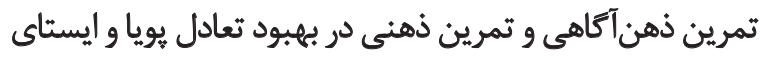

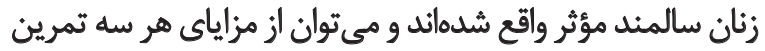

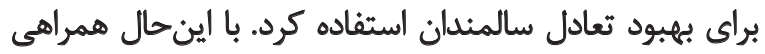

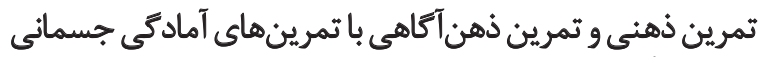

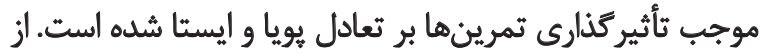

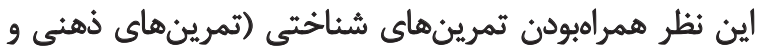

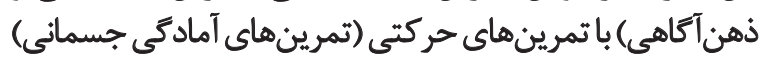
نتايج بايدارترى براى شركت كنيدكان تحقيق ايجاد كرد.

از محدوديتهاى تحقيق حاضر مىتوان به موارد زير اشاره كرد:

محدوديتهاى قابل كنترل (سن و جنسيث آزمودنىها، زمان

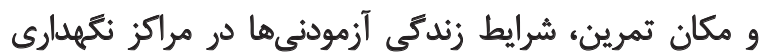

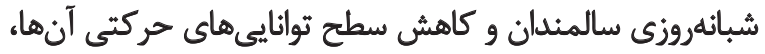

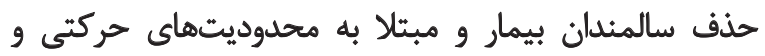

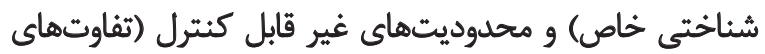

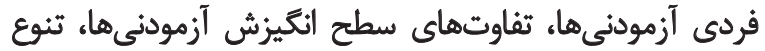

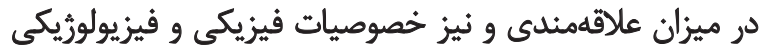

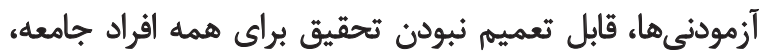
بررسىنكردن جامع و فراكير سالمندان يك شهر و استان و كشور).

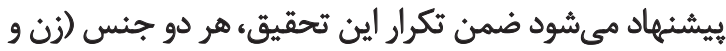

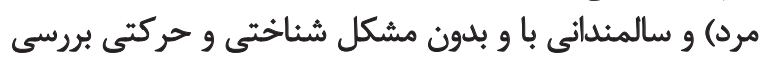

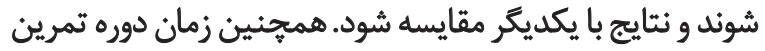

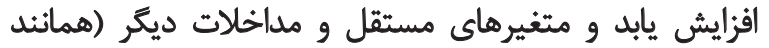

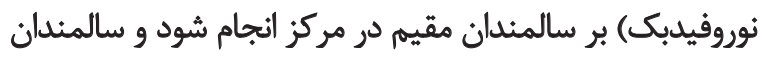
فعال و غيرفعال زن و مرد بالهنان مقيم مقايسه شوكزند.

$$
\text { تشيكر و قدردافى }
$$

اين مقاله بركرفته از باياننامه دكترى لطيفه قاسميور، تروه

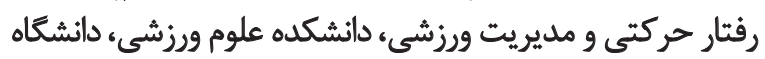

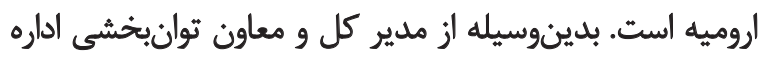




\section{References}

[1] Davarmanesh A, Baratysadeh F. [An introduction to handicaps rehabilitation principles (Persian)]. $1^{\text {st }}$ edition. Tehran: Roshd Publication; 2006.

[2] Gregory Payne V. Human motor development [H. Khalaji, D. Khajavi, Persian trans.]. Arak: Arak University Publication; 2005.

[3] Haywood, K. Lifespan motor development. [M. Namazizadeh, M. Aslankhany, Persian trans.]. $9^{\text {th }}$ edition. Tehran: SAMT; 2008.

[4] Letafetkar A, Abdolvahabi Z. [Advanced corrective exercises (from theory to practice) (Persian)]. Tehran: Avayeh Zohor Publication; 2015.

[5] Sadeghi H, Norouzi H, Karimi Asl A, Montazer M. [Functional training program effect on static and dynamic balance in male able-bodied elderly (Persian)]. Iranian Journal of Ageing. 2008; 3(2):565-71.

[6] Kaufman KA. Evaluating mindfulness as a new approach to athletic performance enhancement. Dissertation Abstracts International: Section B. Sciences and Engineering. 2009; 69(12):7814.

[7] Mohammadi Soreh M. [The effect of mindfulness on Perceived stress and cognitive insight in pregnant women (Persian)] [MSc. thesis]. Urmia: Urmia Branch, Islamic Azad University; 2016.

[8] Holmes PS. The psychophysiology of imagery in sport. Human Kinetics. 2006; 251-261.

[9] Ghasemiyan Moghadam R, Shamspour Dehkordi P, Shams A. [A review of the literature and the mechanisms of mental practice (Persian)]. Journal of Neshat-e Varzesh. 2008; 5(10): 25-31.

[10] Karami M, Hadian MR, Abdolvahab M, Raji P, Yekaninejad S, Montazeri A. [Effects of mental practices on quality of life in elderly men (60-80yrs) (Persian)]. Journal of Modern Rehabilitation. 2014; 8(3):21-32.

[11] Shamsipour-Dehkordy P, Aslankhani MA, Shams A. [Effects of physical, mental and mixed practices on the static and dynamic balance of aged people (Persian)]. Journal of Shahrekord University of Medical Sciences. 2010; 12(4):71-77.

[12] Kee YH, Chatzisarantis NNLD, Kong PW, Chow JY, Chen LH. Mindfulness, Movement Control, and Attentional Focus Strategies: Effects of mindfulness on a postural balance task. Journal of Sport \& Exercise Psychology. 2012; 34(5):561-79. doi: 10.1123/ jsep.34.5.561

[13] Kangasniemi A, Lappalainen R, Kankaanpa A, Tammelin T. Mindfulness skills, psychological flexibility, and psychological symptoms among physically less active and active adults. Mental Health and Physical Activity. 2014; 7(3):121-7. doi: 10.1016/j. mhpa.2014.06.005

[14] Ghavi S, Sohrabi M, Golmohamadi B, Karimi N, Sahaf R, Rahimi M. [The effect of whole body vibration training and mental practice on balance in older men (Persian)]. Journal of Ageing. 2015; 9(4):306-317.

[15] Hosseini SS. The effect of aquatic and mental trainings on balance in elderly males. Middle-East Journal of Scientific Research. 2011, 7(3):296-302.

[16] Baer RA. Mindfulness-based treatment approaches: Clinician's guide to evidence base and appli-cations (practical resources for the mental health professional). Massachusetts: Academic Press; 2014.

[17] Fallah-Pour M, Joghataei MT, A'shayeri H, Salavati M, Hosseini SA. [Effects of mental practice on balance in the elderly (Persian)]. Journal of Rehabilitation. 2003; 4(3, 4):34-9.

[18] Brill PA. Functional fitness for older adults. [N. Rostamy, Persian trans.]. $2^{\text {nd }}$ edition. Tehran: Meayar-e Elm; 2011.

[19] Yim-Chiplis PK, Talbot LA. Defining and measuring balance in adults. Biological Research For Nursing. 2000; 1(4):321-331. doi: $10.1177 / 109980040000100408$

[20] Khasnis A, Gokula RM. Romberg's test. Journal of Postgraduate Medicine. 2003; 49(2):169-172. PMID: 12867698

[21] Davatgaran Tagheipour K. [Translation and standardization of the Persian version of equivalent Berg Balance Scale in the Iranian Elderly (Persian)] [MSc. thesis]. Tehran: University of Welfare and Rehabilitation Sciences; 2005.

[22] Kazemi B, Jazaieri M, Etemadi A. [The effect of balance exercises in the elderly women by rolling boards in sitting position. (Persian)]. Journal of Armaghane-Danesh. 2003; 8(32):23-32.

[23] Berg K, Wood-Dauphinee S, Williams JI, Gayton D. Measuring balance in the elderly: Preliminary development of an instrument. Physiotherapy Canada. 1989; 41(6): 304-311. doi: 10.3138/ ptc.41.6.304

[24] Ordobadi SH. [Practical guide to mindfulness based stress reduction (Persian)] [MSc. thesis]. Urmia: Urmia University; 2016.

[25] Azimzadeh A, Ashlankhani MA, Shojaei M, Salavati M. [Effect of hydrotherapy on static and dynamic balance in older adults: Comparison of per-turbed and non-perturbed programs (Persian)]. Iranian Journal of Ageing. 2013; 7(4):27-34.

[26] Sadeghi H, Alirezaei F. [ The effect of water exercise program on static and dynamic balance in elderly women (Persian)]. Iranian Journal of Ageing. 2008; 2(6):402-409.

[27] Aslankhani M, Farsi A, Sohbatiha M. [The impact of exercise in- and out- water on balance and gait in older men (Persian)] Raftar-e Harekati va Ravanshenasi-ye- Varzesh. 2012; 10:91-104.

[28] Abbasi A, Sadeghi H, Berenjeian Tabrizi H, Bagheri K, Ghasemizad A. [Effects of aquatic balance training and detraining on neuromuscular performance and balance in healthy middle aged male (Persian)]. Koomesh. 2012; 3(13):345-53.

[29] Janati S, Sohrabi M, AtarzadehHoseini SR. [The effect of selective hata yoga training on balance of elderly women (Persian)] Iranian Journal of Ageing. 2010; 5(18):46-52.

[30] Mahdizadeh A, Sadeghi H, Shokhanghoei Y. [The effect of balance-Strength training on balance elderly women with knee osteoarthritis (Persian)]. Paper presented at: The $6^{\text {th }}$ National Congress of Students of Physical Education and Sport Sciences. 2011 December 15-16; Tehran, Iran. 
